\title{
Transfusion, erythropoiesis-stimulating agent therapy, and kidney transplant wait time
}

\author{
Robert M. Perkins ${ }^{1,2 *}$, H. Lester Kirchner ${ }^{3}$, Rajesh Govindasamy ${ }^{2}$ \\ ${ }^{1}$ Center for Health Research, Geisinger Medical Center, Danville, USA \\ ${ }^{2}$ Department of Nephrology, Geisinger Medical Center, Danville, USA \\ ${ }^{3}$ Division of Medicine, Geisinger Medical Center, Danville, USA \\ Email: ${ }^{*}$ rmperkins@geisinger.edu
}

Received 29 June 2011; revised 10 October 2011; accepted 21 October 2011

\begin{abstract}
Aim: Anemia is highly prevalent among patients waitlisted for renal transplant, and management with blood transfusion or erythropoietin stimulating agents may impact transplant wait time. The purpose of this study was to examine the impact of blood transfusion and erythropoiesis stimulating agent therapy on renal transplant wait time. Methods: We retrospectively analyzed all adult patients listed for first deceased donor kidney transplantation at two transplant centers in Central Pennsylvania between 2004 and 2008. The exposures of interest were blood transfusion and erythropoietin stimulating agent therapy. Cox proportional hazards were used to model time to deceased donor kidney transplant. Results: Among 407 patients listed for transplant, 84 received a deceased donor kidney during a median follow-up of 26.3 months. In an adjusted Cox proportional hazards model, with erythropoiesis stimulating agent and transfusion both treated as time-dependent exposures, UNOS inactive status at listing date (hazard ratio [HR] 0.81; 95\% CI $0.73-0.89 ; P<0.001)$ and transfusion during the wait list period (HR 0.27; 95\% CI $0.11-0.69 ; P=0.01$ ) independently predicted longer transplant wait time. Erythropoiesis stimulating agent use prior to or after transplant wait listing date did not independently predict wait time. Conclusion: Blood transfusion while waitlisted for kidney transplant is strongly associated with prolonged wait time.
\end{abstract}

Keywords: Anemia; Blood Transfusion; Erythropoietin; Kidney Disease; Transplantation

\section{INTRODUCTION}

Renal transplantation is the preferred therapy for patients with end-stage renal disease (ESRD) because it is associated with better long-term survival, [1] improved qual-

${ }^{*}$ Corresponding author. ity of life, and lower costs [2]. For potential deceaseddonor renal transplant recipients, time spent on the transplant wait list has increased during the past decade. For those patients listed in 2009, it is expected that median wait time will approach 4 years, an increase of nearly $25 \%$ compared with wait times for patients listed in 2004 [3]. A longer renal transplant wait time-particularly when receiving maintenance hemodialysis - is associated with an increased risk of cardiovascular and other morbidity and shorter graft and patient survival after transplantation $[4,5]$.

Several factors have been consistently associated with longer renal transplant wait times, including non-white race, $\mathrm{B}$ and $\mathrm{O}$ blood types, and immunologic sensitization, as reflected by an elevated panel reactive antibody (PRA) level [6,7]. Along with pregnancy and prior organ transplant, transfusion of red blood cells can contribute to the elevation in PRA among renal transplant candidates [8], which suggests that transfusion might be associated with longer transplant wait time.

Anemia of chronic kidney disease (CKD) is highly prevalent among patients listed for renal transplant. The management of this condition has undergone a major change in the past 20 years, as erythropoiesis-stimulating agent (ESA) therapy has largely replaced intermittent transfusion of packed red blood cells among patients with ESRD. Although the rates of blood transfusion have declined substantially in this population [9], it still remains a common intervention in patients not treated with ESAs [10].

Despite the relatively recent evolution of anemia management among patients with late-stage CKD, the impact of packed red blood cell transfusion or ESA therapy on transplant wait time has not been systematically reported. The goal of this study was to determine that impact among those listed for first deceased donor renal transplant.

\section{METHODS}

This was a retrospective cohort study of CKD patients 
listed for a deceased donor kidney transplant. The study was approved by the Geisinger Medical Center Institutional Review Board in December, 2009 (protocol \# 20100136). The data source was EpicCare, Geisinger Medical Center's electronic health record, which contains detailed demographic, lifestyle (e.g., smoking), procedural, laboratory, radiographic, vital, and other clinical data for all patients receiving care at any of more than 40 outpatient clinics and 3 inpatient facilities, and has been previously used as a research instrument [11].

All patients 18 years or older with stage 4 or 5 CKD and listed for renal transplantation at either of Geisinger's 2 transplant centers between July 1, 2004, and November 30, 2008, were eligible for the study. Patients with a prior solid-organ transplant were excluded, as were those who received a living-donor kidney transplant during the study period. Study outcomes were assessed through November 30, 2009.

Baseline information was obtained during the 6 months before the United Network for Organ Sharing (UNOS) listing date (index date) for each patient. Extracted information included demographic data; detailed comorbid disease history; clinical encounters; laboratory test results; prescription and procedure orders. International Classification of Diseases, version 9 (ICD-9) codes from a minimum of 2 separate outpatient encounters or listed on the medical problem list were used to document the presence of any comorbid condition (CHF: 425.4 - 425.9, 428, 428.**; cerebrovascular disease: 438, 438.0, 438.*0, 438.*1, 438.*2, 438.*9, 438.53, 438.6* $438.9^{*}, 438.6$ - 438.9, 437, 437.0, 437.0 - 437.2, 437.8, 437.9, 436, 435, 435.*, 434, 434.*, 434.**, 433, 433.*, 433.**; diabetes: $250,250 .^{*}, 250 .^{* *}$; pulmonary disease: 491, 491.*, 491.**, 492, 492.*, 493, 493.*, 493.**, 494, 494.*, 496; peripheral vascular disease: 440, 440.*, 440.**, 443, 443.8, 443.9, 443.8*, 445.0*, 445.8*, 557, 557.*). Dialysis, transplant, and vital status were extracted from 6 months prior to index date through the end of the study period (November 30, 2009). Confirmation of renal transplantation was made by linking a cohort member's medical record number from EpicCare with Geisinger's renal transplant service database, and further verified by identifying CPT codes 50360 and 50365 in EpicCare.

The Charlson Comorbidity Index (CCI) [12] score was calculated at baseline for each patient. Baseline laboratory and vital information was recorded using the value closest in time and preceding the transplant listing date. For the purposes of coding maintenance dialysis and intravenous (IV) iron status, outpatient CPT codes for hemodialysis or peritoneal dialysis (90960-63, 90966, $90999,90945,90947)$ and a prescription order for IV iron at any point during the period 6 months before transplant listing date through end of the study period qualified. All laboratory analyses were performed at a single central laboratory located on the campus of the Geisinger Medical Center, with the exception of human leukocyte antigen (HLA) antibody testing, which was performed at a single external contracting laboratory using the Lumine ${ }^{\circledR} 100^{\text {TM }}$ IS Total System analyzer (Luminex, Inc; Austin, TX, USA), with One Lambda PRA microcytotoxicity reagent testing (One Lambda, Inc; Canoga Park, CA, USA). Patients were censored for death, or at the end of the study period.

The primary exposure of interest was packed red blood cell transfusion during the period 6 months before listing date through the end of the study period. For the purpose of providing a descriptive comparison of the group transfused vs. those not transfused (as shown in Table 1), transfusion was treated as a simple binary stratification term (i.e., if ever transfused during the period 6 months prior to listing through the end of the study period, a subject was grouped in that category). For the adjusted, time-to-event analysis, however, cumulative transfusion burden was considered (i.e., each distinct transfusion event was included in the model in additive fashion, and only at the time at which it occurred). In this manner, exposure was defined at the time of transfusion, rather than assuming a fixed exposure at the start of transplant wait time. The number of transfused units at each transfusion occurrence was not considered-each transfusion event was considered equivalent regardless of the total number of units transfused. Transfusion was identified using CPT code 36430 (transfusion of blood or blood components) during the study period, and was confirmed by cross-linking medical record numbers for cohort members with Geisinger's internal blood bank database.

The secondary exposure of interest was any ESA therapy (prescription order for Epoetin alfa or darbepoetin alfa) during the period 6 months before listing date through the end of the study period. As with transfusion, ESA therapy was treated as a time-dependent variable in the Cox proportional hazard model. For ESA, this meant that a patient with a prescription for ESA was treated as "exposed" for a period 12 months after that order date, but unexposed during other periods, if no refill or new order was placed for the medication. In this manner, gaps in ESA therapy were accounted for in the Cox model.

The primary study outcome was transplant wait time, defined as the time in months from index date to date of deceased donor kidney transplantation.

\section{STATISTICAL ANALYSIS}

Descriptive statistics, including mean and standard deviation (SD) for continuous variables and frequency and percentage for categorical variables, were presented for 
Table 1. Baseline characteristics of patients listed for first deceased donor renal transplant, by blood transfusion status ${ }^{\dagger}$.

\begin{tabular}{|c|c|c|c|}
\hline & No Transfusion $(n=336)$ & Transfusion $(n=71)$ & P-value \\
\hline Mean (SD) age at listing date, years & $53.3(13.3)$ & $54.8(11.4)$ & 0.36 \\
\hline Male sex, n (\%) & $202(60.1)$ & $46(64.8)$ & 0.46 \\
\hline White race, $\mathrm{n}(\%)$ & $307(91.4)$ & $63(88.7)$ & 0.48 \\
\hline Mean (SD) BMI, $\mathrm{kg} / \mathrm{m}^{2}$ & $29.0(6.1)$ & $30.5(6.6)$ & 0.07 \\
\hline Blood type, n (\%) & & & 0.51 \\
\hline A & $136(40.5)$ & $35(49.3)$ & \\
\hline $\mathrm{AB}$ & $16(4.8)$ & $2(2.8)$ & \\
\hline B & $38(11.3)$ & $10(14.1)$ & \\
\hline $\mathrm{O}$ & $144(42.9)$ & $24(33.8)$ & \\
\hline Inactive UNOS status at listing, $\mathrm{n}(\%)$ & $282(90.1)$ & $64(92.8)$ & 0.49 \\
\hline Charlson Comorbidity Index score, n (\%) & & & 0.04 \\
\hline 0 & $265(78.9)$ & $46(64.8)$ & \\
\hline 1 & $32(9.5)$ & $11(15.5)$ & \\
\hline$\geq 2$ & $39(11.6)$ & $14(19.7)$ & \\
\hline Congestive heart failure, $\mathrm{n}(\%)$ & $10(3.0)$ & $3(4.2)$ & 0.71 \\
\hline Cerebrovascular disease, $\mathrm{n}(\%)$ & $7(2.1)$ & $3(4.2)$ & 0.39 \\
\hline Diabetes, n (\%) & $43(12.8)$ & $16(22.5)$ & 0.03 \\
\hline Pulmonary disease, $\mathrm{n}(\%)$ & $14(4.2)$ & $3(4.2)$ & 0.9 \\
\hline Peripheral vascular disease, $\mathrm{n}(\%)$ & $12(3.6)$ & $3(4.2)$ & 0.73 \\
\hline Mean (SD) hemoglobin level, g/dL & $12.3(1.8)$ & $12.1(1.8)$ & 0.36 \\
\hline Mean (SD) GFR, ml/min/1.73 $\mathrm{m}^{2 \dagger \dagger}$ & $15.5(5.6)$ & $14.2(5.2)$ & 0.28 \\
\hline Peak PRA (from listing date to end of follow up) ${ }^{\S}$ & & & 0.77 \\
\hline $0 \%-9 \%, \mathrm{n}(\%)$ & $129(38.39)$ & $24(33.80)$ & \\
\hline $10 \%-79 \%, \mathrm{n}(\%)$ & $36(10.71)$ & $6(8.45)$ & \\
\hline$\geq 80 \%, \mathrm{n}(\%)$ & $19(5.65)$ & $5(7.04)$ & \\
\hline Dialysis, n (\%) & $206(61.3)$ & $42(59.2)$ & 0.74 \\
\hline IV iron, $\mathrm{n}(\%)$ & $22(6.6)$ & $5(7.0)$ & 0.8 \\
\hline ESA use in prior 6 months, $\mathrm{n}(\%)$ & $80(23.8)$ & $22(31.0)$ & 0.21 \\
\hline
\end{tabular}

$\mathrm{BMI}=$ body mass index; ESA = erythropoiesis-stimulating agent; GFR = glomerular filtration rate; IV = intravenous; UNOS = United Network for Organ Sharing. ${ }^{\dagger}$ Transfusion window includes the 6-month period before the transplant listing date through the end of the study period. Lab values and weights were results closest to listing date. ${ }^{\dagger \dagger}$ Reported for subjects not on dialysis at time of transplant listing. ${ }^{\S}$ Data missing for $47.2 \%$ of study population.

the study sample stratified by transfusion status. Demographic and medical characteristics were compared between the two groups using the 2-sample $t$ - and Pearson chi-square tests as appropriate. For descriptive purposes, Kaplan-Meier curves were presented estimating the cumulative incidence of transplant for the two groups.

Cox proportional hazard regression models were used to estimate the adjusted associations with transplant wait time. First, univariate regression models were employed. Variables from the univariate analysis associated with the outcome at $P<0.10$ or that were plausibly associated with transplant wait time were included in a full multivariate Cox regression model to account for possible confounding effects. In the Cox model, both transfusion and ESA were treated as two distinct temporal exposures-therapy during the 6-months prior to listing date, 
and therapy after the listing date. The results of the models were expressed as hazard ratios (HRs) with 95\% CIs. A HR greater than one meant the corresponding variable was associated with higher probability of receiving a transplant, hence a shorter transplant wait time. SAS ${ }^{\circledR}$ Version 9.2 (SAS Corporation, Cary, NC, USA) was used for all analyses.

\section{RESULTS}

During the study period, 485 patients were listed for first renal transplantation. Of these, 78 received a living donor kidney and were excluded, leaving 407 patients in the final study cohort. The median follow up period was 26.3 months, during which 84 patients received a deceased donor renal transplant. 71 patients $(17 \%)$ received a total of 162 transfusions during the study period; 7 patients received a total of 8 transfusions during the 6 month period prior to transplant listing, and 67 patients received a total of 154 transfusions after the listing date. Of those transfused after the listing date, 45 (67\%) received 2 or fewer units, while $22(33 \%)$ received 3 or more.

At baseline, differences were minimal between those who received transfusions and those who did not (Table 1). Patients who received a transfusion at any point during the study period were more likely to have diabetes and a greater co-morbid disease burden than patients who did not. ESA use prior to transplant listing was similar between those who did and did not receive a transfusion. There was no statistically significant difference observed in peak PRA levels during the study period between the 2 groups in patients with PRA data available. Because approximately half of the overall study population had no recorded PRA level during the study period, this covariate was not included in adjusted models.

Among patients who received a transfusion at any time during the study period, $4(5.6 \%)$ received a deceased donor kidney transplant during the study period, compared with $80(23.8 \%)$ patients who had no transfusion. Twenty-three $(32.4 \%)$ deaths occurred while awaiting renal transplantation in the transfusion group; 69 $(20.5 \%)$ deaths occurred in the no-transfusion group $(P=$ $0.03)$.

In the unadjusted analyses examining the association with time to first renal transplant, higher body mass in$\operatorname{dex}(\mathrm{HR}=0.96 ; 95 \% \mathrm{CI}, 0.93-0.99 ; P=0.02)$, inactive UNOS status at time of listing ( $\mathrm{HR}=0.83 ; 95 \% \mathrm{CI}, 0.75$ - 0.91; $P<0.001$ ), and transfusion of packed red blood cells during the wait list period $(\mathrm{HR}=0.18 ; 95 \% \mathrm{CI}$, $0.04-0.48 ; P<0.001)$ were significantly associated with longer transplant wait time. Age, gender, race, weight, blood type, CCI score, co-morbid conditions, baseline hemoglobin level, baseline glomerular filtration rate, baseline dialysis status, prior ESA use, and peak PRA levels were not statistically significant predictors.

In the multivariate Cox proportional hazard regression analysis, independent predictors of longer transplant wait time included baseline inactive UNOS listing status and transfusion of packed red blood cells after the date of listing, but not within 6 months prior to the listing date (Table 2). Exposure to ESA prior to the listing date (HR $0.82,95 \%$ CI $0.44-1.56, P=0.55)$ or during the wait list period (HR $0.88,95 \%$ CI $0.45-1.72, P=0.7$ ) was not significantly associated with time to first deceased donor renal transplant. Co-linearity (between ESA exposure prior to and after waitlisting, and similarly between transfusion status prior to and after waitlisting) was assessed. These analyses were nonsignificant. In addition, as ESA use may be more likely in those requiring a blood transfusion, the Cox model was repeated after first excluding anyone who was transfused $(\mathrm{n}=71)$; this did not substantially alter the association between ESA use and transplant wait time (data not shown).

Table 2. Multivariate cox proportional hazard analysis on time to first deceased donor renal transplant $(\mathrm{N}=407)$.

\begin{tabular}{cccc}
\hline & Hazard Ratio & 95\% CI & P-value \\
\hline Inactive UNOS status at listing date & 0.81 & $0.73-0.89$ & $<.001$ \\
Blood transfusion (during 6-month period prior to listing date) & 0.59 & $0.14-2.46$ & 0.47 \\
Blood transfusion (after listing date), per unit transfused & 0.27 & $0.11-0.69$ & 0.01 \\
ESA (during 6-month period prior to listing date) & 0.82 & $0.44-1.56$ & 0.5 \\
ESA (after listing date) & 0.88 & $0.45-1.72$ & 0.1 \\
Dialysis & 0.65 & $0.39-1.09$ & 0.09 \\
IV iron & 2.23 & $0.904-5.017$ & 0.08 \\
\hline
\end{tabular}

IV = intravenous; UNOS = United Network for Organ Sharing. Model adjusted for age, race, BMI at listing date, Charlson Comorbidity Index score, and ABO blood group. ESA status and cumulative transfusion status were time-adjusted as described in the methods. 


\section{DISCUSSION}

In this health system analysis, blood transfusion after listing for renal transplant was strongly associated with prolonged renal transplant wait time; patients who received a transfusion after the listing date were more than $70 \%$ less likely to receive a deceased donor renal transplant for each unit transfused, compared with those who were not transfused. There was a trend towards longer transplant wait time among those transfused during the 6 months prior to wait list date which was not significant, potentially because of the relatively small number of transfusions $(\mathrm{n}=8)$ occurring among the cohort during this time period.

The relationship between blood transfusion and renal transplant outcomes is complex and controversial. Historically, pre-transplant transfusion was thought to confer a graft-protective effect via induction of immunologic tolerance [13]. The introduction of improved immunosuppressive regimens - specifically calcineurin inhibitor-based regimens - was thought to negate this potential beneficial effect [14], and the practice has been largely abandoned. However, studies examining this question have not addressed the impact of transfusion on transplant wait time, the focus of our study.

While the impact of transfusion on PRA levels has been studied, [15] and the association between higher PRA levels and delayed renal transplantation is wellrecognized [6,7], to our knowledge no prior study has directly assessed the association between transfusion of packed red blood cells and renal transplant wait time. Although our study cannot demonstrate causality, the association between transfusion after listing date and prolonged wait time remained strong despite the inclusion of other covariates that might be associated with prolonged transplant wait time.

ESA therapy was not independently associated with shorter transplant wait time in this analysis. There are multiple potential explanations for this observation. ESAs are not immunologically inert; varying effects of this class of drugs on human T-cell function and cytokine signaling have been reported $[16,17]$. However, the net effect of the cytokine-modulating actions of these drugs on immune profiles is not clear.

Second, any potential beneficial impact of ESAs on transplant wait time might be limited to those with higher peak PRA levels, a subgroup of patients who are underrepresented in our analysis. Several older, small studies have examined the impact of ESA therapy on PRA levels among ESRD patients [18-20]. As a whole, these studies would suggest the impact of ESA therapy on PRA levels is modest, and perhaps limited to those most highly sensitized [18-20]. The fact that this study cohort included very few patients with peak PRA levels $>80 \%$ might have limited our ability to demonstrate that impact.

Finally, the effects of confounding by ESA indication not accounted for in the analysis should be considered. ESA therapy occurred more frequently among those who received transfusions than those who did not, though the difference was not significant. In addition, the effect estimates for ESA in the Cox model were qualitatively no different after excluding those who were transfused from the analysis.

These findings have potential clinical implications. In an era of heightened ESA sensitivity due to concerns about the cardiovascular risks of this medication class, an increase in transfusions among patients with late stage 4 CKD might be expected. While our findings do not establish causality between transfusion and longer wait time, increased reliance upon transfusion as an anemia management strategy among this population may prolong wait time for listed patients. Further, our findings would not suggest that ESA therapy as a transfusionavoidance intervention would shorten time spent on the wait list. Therefore, as with trends in anemia management generally among patients with advanced CKD, tolerance of a lower hemoglobin level for the appropriate waitlisted patient may be the approach with fewest negative potential consequences. When transfusion is required, use of leukopore-filtered blood products may limit the potential immunologic impact.

This study has limitations. Because a subset of the patient population received maintenance hemodialysis outside the Geisinger system, data regarding medication use and laboratory tests may be incomplete for these patients; therefore, misclassification bias may have occurred. Although the multivariate model included covariates that might be associated with transplant waiting time and transfusion, the potential for residual confounding in this cohort study persists, particularly for factors related to unobserved comorbid disease severity. Additional limitations include limited data on PRA levels for study participants: PRA results were obtained for all patients who received blood work, but not all study participants - in fact nearly half of the population-had these tests performed after the index date, likely because of the relatively large numbers of patients listed as inactive at the time of transplant listing. Finally, the predominantly white study population reflects the demographic profile of central Pennsylvania but is not representative of the overall US population with advanced CKD, thus limiting the generalizability of the study findings to more racially diverse populations.

In conclusion, transfusion of packed red blood cells after listing for renal transplant strongly correlates with prolonged transplant wait time among patients listed for first deceased donor renal transplant. 


\section{ACKNOWLEDGEMENTS}

The investigators thank Amanda Bengier and Ryan Kissinger for assistance with data extraction and programming; Haiyan Sun for assistance with data analysis; and Zahra Daar, Bradley Moyer, Dikran Toroser, and Margit Rezabek for editorial assistance. Dr. Perkins has received research funding and honoraria from American Regent. Dr. Kirchner has received research funding from American Regent. This project was funded by Amgen, Inc.

\section{REFERENCES}

[1] Port, F.K., Wolfe, R.A., Mauger, E.A., Berling, D.P. and Jiang, K. (1993) Comparison of survival probabilities for dialysis patients vs cadaveric renal transplant recipients. Journal of the American Medical Association, 270, 13391343. doi:10.1001/jama.270.11.1339

[2] Laupacis, A., Keown, P., Pus, N., Krueger, H., Ferguson, B., Wong, C. and Muirhead, N. (1996) A study of the quality of life and cost-utility of renal transplantation. Kidney International, 50, 235-242. doi:10.1038/ki.1996.307

[3] Danovitch, G.M., Cohen, D.J., Weir, M.R., Stock, P.G., Bennet, W.M., Christensen, L.L. and Sung, R.S. (2005) Current status of kidney and pancreas transplantation in the United States, 1994-2003. American Journal of Transplantation, 5, 904-915. doi:10.1111/j.1600-6135.2005.00835.x

[4] Meier-Kriesche, H.U. and Kaplan, B. (2002) Waiting time on dialysis as the strongest modifiable risk factor for renal transplant outcomes: A paired donor kidney analysis. Transplantation, 74, 1377-1381. doi:10.1097/00007890-200211270-00005

[5] Mange, K.C., Joffe, M.M. and Feldman, H.I. (2001) Effect of the use or nonuse of long-term dialysis on the subsequent survival of renal transplants from living donors. The New England Journal of Medicine, 344, 726731. doi:10.1056/NEJM200103083441004

[6] Sanfilippo, F.P., Vaughn, W.K., Peters, T.G., Shield, C.F. 3rd, Adams, P.L., Lorber, M.I. and Williams, G.M. (1992) Factors affecting the waiting time of cadaveric kidney transplant candidates in the United States. Journal of the American Medical Association, 267, 247-252. doi:10.1001/jama.1992.03480020057031

[7] Wolfe, R.A., Ashby, V.B., Milford, E.L., Bloembergen, W.E., Agodoa, L.Y.C., Held, P.J. and Port, F.K. (2000) Differences in access to cadaveric renal transplantation in the United States. American Journal of Kidney Diseases, 36, 1025-1033. doi:10.1053/ajkd.2000.19106

[8] Vella, J.P., O’Neill, D., Atkins, N., Donohoe, J.F. and Walshe, J.J. (1998) Sensitization to human leukocyte antigen before and after the introduction of erythropoietin. Nephrology Dialysis Transplantation, 13, 2027-2032. doi:10.1093/ndt/13.8.2027

[9] Ibrahim, H.N., Ishani, A., Foley, R.N., Guo, H., Liu, J. and Collins, A.J. (2008) Temporal trends in red blood transfusion among US dialysis patients, 1992-2005. American Journal of Kidney Diseases, 52, 1115-1121. doi:10.1053/j.ajkd.2008.07.022

[10] Lawler, E.V., Bradbury, B.D., Fonda, J.R., Gaziano, J.M. and Gagnon, D.R. (2010) Transfusion burden among patients with chronic kidney disease and anemia. Clinical Journal of the American Society of Nephrology, 5, 667672. doi:10.2215/CJN.06020809

[11] Wu, J., Roy, J. and Stewart, W.F. (2010) Prediction modeling using EHR data: Challenges, strategies, and a comparison of machine learning approaches. Medical Care, 48, 106-113. doi:10.1097/MLR.0b013e3181de9e17

[12] Charlson, M.E., Pompei, P., Ales, K.L. and MacKenzie, C.R. (1987) A new method of classifying prognostic comorbidity in longitudinal studies: Development and validation. Journal of Chronic Diseases, 40, 373-383. doi:10.1016/0021-9681(87)90171-8

[13] Van Es, A.A. and Balner, H. (1979) Effect of pretransplant transfusions on kidney allograft survival. Transplantation Proceedings, 11, 127-137.

[14] Lundgren, G., Groth, C.G., Albrechtsen, D., Brynger, H., Flatmark, A., Frödin, L., Gäbel, H., Husberg, B., Klintmalm, G. and Maurer, W. (1986) HLA-matching and pretransplant blood transfusions in cadaveric renal transplantation-A changing picture with cyclosporin. Lancet, 2, 66-69. doi:10.1016/S0140-6736(86)91608-9

[15] Sanfilippo, F., Vaughn, W.K., Bollinger, R.R. and Spees, E.K. (1982) Comparative effects of pregnancy, transfusion, and prior graft rejection on sensitization and renal transplant results. Transplantation, 34, 360-366. doi:10.1097/00007890-198212000-00010

[16] Kristal, B., Shurtz-Swirski, R., Shasha, S.M., Manaster, J., Shapiro, G., Furmanov, M., Hassan, K., Weissman, I. and Sela, S. (1999) Interaction between erythropoietin and peripheral polymorphonuclear leukocytes in hemodialysis patients. Nephron, 81, 406-413. doi: $10.1159 / 000045324$

[17] Strunk, T., Hartel, C., Temming, P., Matzke, N., Zimmer, J. and Schultz, C. (2008) Erythropoietin inhibits cytokine production of neonatal and adult leukocytes. Acta Paediatrica, 97, 16-20. doi:10.1111/j.1651-2227.2007.00560.x

[18] Grimm, P.C., Sinai-Trieman, L., Sekiya, N.M., Robertson, L.S., Robinson, B.J., Fine, R.N. and Ettenger, R.B. (1990) Effects of recombinant human erythropoietin on HLA sensitization and cell mediated immunity. Kidney International, 38, 12-18. doi:10.1038/ki.1990.161

[19] Koskimies, S., Lautenschlager, I., Gronhagen-Riska, C. and Hayry, P. (1990) Erythropoietin therapy and the antibody levels of highly sensitized patients awaiting kidney transplantation. Transplantation, 50, 707-709. doi:10.1097/00007890-199010000-00035

[20] Grimm, P.C., Sekiya, N.M., Robertson, L.S., Robinson, B.J. and Ettenger, R.B. (1991) Recombinant human erythropoietin decreases anti-HLA sensitization and may improve renal allograft outcome: Involvement of antiidiotypic antibody. Transplantation Proceedings, 23, $407-$ 408. 